\title{
On quantum cluster algebras of finite type
}

\author{
Ming Ding \\ Institute for advanced study, Tsinghua University, Beijing 100084, China
}

\begin{abstract}
We extend the definition of a quantum analogue of the CalderoChapoton map defined [17. When $Q$ is a quiver of finite type, we prove that the algebra $\mathscr{A} \mathscr{H}_{|k|}(Q)$ generated by all cluster characters (see Definition 11) is exactly the quantum cluster algebra $\mathscr{E} \mathscr{H}_{|k|}(Q)$.
\end{abstract}

Keywords cluster variable, quantum cluster algebra MSC 16G20

\section{Introduction}

Quantum cluster algebras were introduced by A. Berenstein and A. Zelevinsky [3] to study the canonical basis. When $q=1$, the quantum cluster algebras are exactly the corresponding cluster algebras which were introduced and studied by S. Fomin and A. Zelevinsky in a series of papers [9] 10]. A quantum analogue of the Caldero-Chapoton formula [4 was defined by D. Rupel [17] and the author conjectured that cluster variables could be expressed using this formula and proved it for the cluster variables in finite types as well as in almost acyclic clusters. Later this conjecture was confirmed for acyclic equally valued quivers in [16]. Quantum cluster algebra structures have been studied in a few cases, see for example [13] 17] 15] 6] [16] 7].

The cluster category was introduced for its combinatorial similarities with cluster algebras. In contrast to the case of cluster algebras, for any objects $M, N$ in the cluster category associated to a quantum cluster algebra, it does not generally hold that $X_{N} X_{M}=|k|^{ \pm \frac{1}{2} n_{N \oplus M}} X_{N \oplus M}$ for any $n_{N \oplus M} \in \mathbb{Z}$. Thus the natural problem is to ask if $X_{N \oplus M}$ is in the corresponding quantum cluster algebra. Hence it becomes interesting to study the relation between the algebra generated by all cluster characters (see Definition 1) and the corresponding quantum cluster algebra. In the case of cluster algebras, these are equal for finite and affine types [5] 8]. In [11]12, C. Geiss, B. Leclerc and J. Schröer have proved that a large class of cluster algebras always contain cluster characters of all objects in the cluster categories. The aim of this article is to prove that for any quiver $Q$ of finite type, the algebra $\mathscr{A} \mathscr{H}_{|k|}(Q)$ generated by all cluster characters is still the quantum cluster algebra $\mathscr{E} \mathscr{H}|k|(Q)$.

E-mail: m-ding04@mails.tsinghua.edu.cn 


\section{Preliminaries and statement of the main result}

2.1 Definition of quantum cluster algebras Let $L$ be a lattice of rank $m$ and $\Lambda: L \times L \rightarrow \mathbb{Z}$ a skew-symmetric bilinear form. Note that $\Lambda$ can be identified with an $m \times m$ skew-symmetric matrix which still denoted by $\Lambda$ if there is no confusion. Set a formal variable $q$ and the ring of integer Laurent polynomials $\mathbb{Z}\left[q^{ \pm 1 / 2}\right]$. Define the based quantum torus associated to the pair $(L, \Lambda)$ to be the $\mathbb{Z}\left[q^{ \pm 1 / 2}\right]$-algebra $\mathscr{T}$ with a distinguished $\mathbb{Z}\left[q^{ \pm 1 / 2}\right]$-basis $\left\{X^{e}: e \in L\right\}$ and the multiplication

$$
X^{e} X^{f}=q^{\Lambda(e, f) / 2} X^{e+f} .
$$

It is known that $\mathscr{T}$ is contained in its skew-field of fractions $\mathscr{F}$. A toric frame in $\mathscr{F}$ is a map $M: \mathbb{Z}^{m} \rightarrow \mathscr{F} \backslash\{0\}$ given by

$$
M(\mathbf{c})=\varphi\left(X^{\eta(\mathbf{c})}\right)
$$

where $\varphi$ is an automorphism of $\mathscr{F}$ and $\eta: \mathbb{Z}^{m} \rightarrow L$ is an isomorphism of lattices. By the definition, the elements $M(\mathbf{c})$ form a $\mathbb{Z}\left[q^{ \pm 1 / 2}\right]$-basis of the based quantum torus $\mathscr{T}_{M}:=\varphi(\mathscr{T})$ and satisfy the following relations:

$$
\begin{gathered}
M(\mathbf{c}) M(\mathbf{d})=q^{\Lambda_{M}(\mathbf{c}, \mathbf{d}) / 2} M(\mathbf{c}+\mathbf{d}), M(\mathbf{c}) M(\mathbf{d})=q^{\Lambda_{M}(\mathbf{c}, \mathbf{d})} M(\mathbf{d}) M(\mathbf{c}), \\
M(\mathbf{0})=1, M(\mathbf{c})^{-1}=M(-\mathbf{c})
\end{gathered}
$$

where $\Lambda_{M}$ is the skew-symmetric bilinear form on $\mathbb{Z}^{m}$ obtained from the lattice isomorphism $\eta$. Let $\Lambda_{M}$ be the skew-symmetric $m \times m$ matrix defined by $\lambda_{i j}=\Lambda_{M}\left(e_{i}, e_{j}\right)$ where $\left\{e_{1}, \ldots, e_{m}\right\}$ is the standard basis of $\mathbb{Z}^{m}$. Given a toric frame $M$, let $X_{i}=M\left(e_{i}\right)$. Then we have

$$
\mathscr{T}_{M}=\mathbb{Z}\left[q^{ \pm 1 / 2}\right]\left\langle X_{1}^{ \pm 1}, \ldots, X_{m}^{ \pm 1}: X_{i} X_{j}=q^{\lambda_{i j}} X_{j} X_{i}\right\rangle
$$

An easy computation shows that:

$$
M(\mathbf{c})=q^{\frac{1}{2} \sum_{i<j} c_{i} c_{j} \lambda_{j i}} X_{1}^{c_{1}} X_{2}^{c_{2}} \cdots X_{m}^{c_{m}}=: X^{(\mathbf{c})} \quad\left(\mathbf{c} \in \mathbb{Z}^{m}\right) .
$$

Let $\Lambda$ be an $m \times m$ skew-symmetric matrix and $\tilde{B}$ an $m \times n$ matrix with $n \leq m$. We call the pair $(\Lambda, \tilde{B})$ compatible if up to permuting rows and columns $\tilde{B}^{T} \Lambda=(D \mid 0)$ with $D=\operatorname{diag}\left(d_{1}, \cdots, d_{n}\right)$ where $d_{i} \in \mathbb{N}$ for $1 \leq i \leq n$. The pair $(M, \tilde{B})$ is called a quantum seed if the pair $\left(\Lambda_{M}, \tilde{B}\right)$ is compatible. Define the $m \times m$ matrix $E=\left(e_{i j}\right)$ as follows

$$
e_{i j}= \begin{cases}\delta_{i j} & \text { if } j \neq k \\ -1 & \text { if } i=j=k \\ \max \left(0,-b_{i k}\right) & \text { if } i \neq j=k\end{cases}
$$


For $n, k \in \mathbb{Z}, k \geq 0$, denote $\left[\begin{array}{l}n \\ k\end{array}\right]_{q}=\frac{\left(q^{n}-q^{-n}\right) \cdots\left(q^{n-k+1}-q^{-n+k-1}\right)}{\left(q^{k}-q^{-k}\right) \cdots\left(q-q^{-1}\right)}$. Let $k \in[1, n]$ where $[1, n]=\{1, \cdots, n\}$ and $\mathbf{c}=\left(c_{1}, \ldots, c_{m}\right) \in \mathbb{Z}^{m}$ with $c_{k} \geq 0$. Define the toric frame $M^{\prime}: \mathbb{Z}^{m} \rightarrow \mathscr{F} \backslash\{0\}$ as follows

$$
M^{\prime}(\mathbf{c})=\sum_{p=0}^{c_{k}}\left[\begin{array}{c}
c_{k} \\
p
\end{array}\right]_{q^{d_{k} / 2}} M\left(E \mathbf{c}+p \mathbf{b}^{k}\right), \quad M^{\prime}(-\mathbf{c})=M^{\prime}(\mathbf{c})^{-1} .
$$

where the vector $\mathbf{b}^{k} \in \mathbb{Z}^{m}$ is the $k$ th column of $\tilde{B}$. Following [9], we say a real $m \times n$ matrix $\tilde{B}^{\prime}$ is obtained from $\tilde{B}$ by matrix mutation in direction $k$ if the entries of $\tilde{B}^{\prime}$ are given by

$$
b_{i j}^{\prime}= \begin{cases}-b_{i j} & \text { if } i=k \text { or } j=k \\ b_{i j}+\frac{\left|b_{i k}\right| b_{k j}+b_{i k}\left|b_{k j}\right|}{2} & \text { otherwise. }\end{cases}
$$

Then the quantum seed $\left(M^{\prime}, \tilde{B}^{\prime}\right)$ is defined to be the mutation of $(M, \tilde{B})$ in direction $k$. Two quantum seeds are called mutation-equivalent if they can be obtained from each other by a sequence of mutations. Let $\mathscr{C}=\left\{M^{\prime}\left(e_{i}\right): i \in[1, n]\right\}$ where $\left(M^{\prime}, \tilde{B}^{\prime}\right)$ is mutation-equivalent to $(M, \tilde{B})$. The elements of $\mathscr{C}$ are called the cluster variables. Let $\mathbb{P}=\left\{M\left(e_{i}\right): i \in[n+1, m]\right\}$ and the elements of $\mathbb{P}$ are called coefficients. Denote by $\mathbb{Z} \mathbb{P}$ the ring of Laurent polynomials generated by $q^{\frac{1}{2}}, \mathbb{P}$ and their inverses. Then the quantum cluster algebra $\mathscr{A}_{q}\left(\Lambda_{M}, \tilde{B}\right)$ is defined to be the $\mathbb{Z} \mathbb{P}$-subalgebra of $\mathscr{F}$ generated by $\mathscr{C}$.

2.2 The quantum Caldero-Chapoton map and main result Let $k$ be a finite field with cardinality $|k|=q$ and $m \geq n$ be two positive integers and $\widetilde{Q}$ an acyclic valued quiver with vertex set $\{1, \ldots, m\}$. Denote the subset $\{n+1, \ldots, m\}$ by $C$. The full subquiver $Q$ on the vertices $1, \ldots, n$ is called the principal part of $\widetilde{Q}$. For $1 \leq i \leq m$, let $S_{i}$ be the $i$ th simple module for $k \widetilde{Q}$.

Let $\widetilde{B}$ be the $m \times n$ matrix associated to the quiver $\widetilde{Q}$ whose entry in position $(i, j)$ given by

$$
\left.b_{i j}=\mid\{\text { arrows } i \longrightarrow j\}|-| \text { arrows } j \longrightarrow i\right\} \mid
$$

for $1 \leq i \leq m, 1 \leq j \leq n$. Denote by $\widetilde{I}$ the left $m \times n$ submatrix of the identity matrix of size $m \times m$. Assume that there exists some antisymmetric $m \times m$ integer matrix $\Lambda$ such that

$$
\Lambda(-\widetilde{B})=\left[\begin{array}{c}
I_{n} \\
0
\end{array}\right]
$$

where $I_{n}$ is the identity matrix of size $n \times n$. Let $\widetilde{R}=\widetilde{R}_{\widetilde{Q}}$ be the $m \times n$ matrix with its entry in position $(i, j)$ given by

$$
\widetilde{r}_{i j}:=\operatorname{dim}_{k} \operatorname{Ext}_{k \widetilde{Q}}^{1}\left(S_{j}, S_{i}\right)=\mid\{\text { arrows } j \longrightarrow i\} \mid .
$$


for $1 \leq i \leq m, 1 \leq j \leq n$. Set $\widetilde{R}^{t r}=\widetilde{R}_{\widetilde{Q}^{o p}}$. Denote the principal $n \times n$ submatrices of $\widetilde{B}$ and $\widetilde{R}$ by $B$ and $R$ respectively. Note that $\widetilde{B}=\widetilde{R}^{t r}-\widetilde{R}$ and $B=R^{t r}-R$.

Let $\mathscr{C}_{\widetilde{Q}}$ be the cluster category of $k \widetilde{Q}$, i.e., the orbit category of the derived category $\mathscr{D}^{b}(\widetilde{Q})$ under the action of the functor $F=\tau$ ०[-1] (see [2]). Let $I_{i}$ be the indecomposable injective $k \widetilde{Q}$ module for $1 \leq i \leq m$. Then the indecomposable $k \widetilde{Q}$-modules and $I_{i}[-1]$ for $1 \leq i \leq m$ exhaust all indecomposable objects of the cluster category $\mathscr{C}_{\widetilde{Q}}$. Each object $M$ in $\mathscr{C}_{\widetilde{Q}}$ can be uniquely decomposed as

$$
M=M_{0} \oplus I_{M}[-1]
$$

where $M_{0}$ is a module and $I_{M}$ is an injective module.

The Euler form on $k \widetilde{Q}$-modules $M$ and $N$ is given by

$$
\langle M, N\rangle=\operatorname{dim}_{k} \operatorname{Hom}(M, N)-\operatorname{dim}_{k} \operatorname{Ext}^{1}(M, N) .
$$

Note that the Euler form only depends on the dimension vectors of $M$ and $N$.

The quantum Caldero-Chapoton map of an acyclic quiver $\widetilde{Q}$ has been defined in [17] and [16]. In [17], the author defined the quantum Caldero-Chapoton map for $k \widetilde{Q}$-modules while in [16] for coefficient-free rigid object in $\mathscr{C}_{\widetilde{Q}}$. For our purpose, we need to extend these definitions to the following map

$$
X_{?}: \operatorname{obj} \mathscr{C}_{\widetilde{Q}} \longrightarrow \mathscr{T}
$$

defined by the following rules:

(1) If $M$ is a $k Q$-module, then

$$
X_{M}=\sum_{\underline{e}}\left|\operatorname{Gr}_{\underline{e}} M\right| q^{-\frac{1}{2}\langle\underline{e}, \underline{m}-\underline{e}-\underline{i}\rangle} X^{-\widetilde{B} \underline{e}-\left(\widetilde{I}-\widetilde{R}^{t r}\right) \underline{m}} ;
$$

(2) If $M$ is a $k Q$-module and $I$ is an injective $k \widetilde{Q}$-module, then

$$
X_{M \oplus I[-1]}=\sum_{\underline{e}}\left|\operatorname{Gr}_{\underline{e}} M\right| q^{-\frac{1}{2}\langle\underline{e}, \underline{m}-\underline{e}-\underline{i}\rangle} X^{-\widetilde{B} \underline{e}-\left(\widetilde{I}-\widetilde{R}^{t r}\right) \underline{m}+\underline{\operatorname{dim}} s o c I},
$$

where $\underline{\operatorname{dim}} I=\underline{i}, \underline{\operatorname{dim}} M=\underline{m}$ and $\operatorname{Gr}_{\underline{e}} M$ denotes the set of all submodules $V$ of $M$ with $\underline{\operatorname{dim}} V=\underline{e}$. We note that

$$
X_{P[1]}=X_{\tau P}=X \underline{\operatorname{dim} P} / \mathrm{rad} P=X \underline{\operatorname{dimsoc} I}=X_{I[-1]}=X_{\tau^{-1} I} .
$$

for any projective $k \widetilde{Q}$-module $P$ and injective $k \widetilde{Q}$-module $I$ with $\operatorname{soc} I=P / \operatorname{rad} P$. In the following, we denote by the corresponding underlined lower case letter $\underline{x}$ the dimension vector of a $k Q$-module $X$ and view $\underline{x}$ as a column vector in $\mathbb{Z}^{n}$.

Definition 1. $X_{L}$ is called the corresponding cluster character, if $L$ is a $k Q$ module or $L=M \oplus I[-1] \in \mathscr{C}_{\widetilde{Q}}$ satisfying that $M$ is a $k Q$-module and $I$ is an injective $k \widetilde{Q}$-module. 
For a quiver $Q$, denote by $\mathscr{A} \mathscr{H}|k|(Q)$ the $\mathbb{Z} \mathbb{P}$-subalgebra of $\mathscr{F}$ generated by all the cluster characters and by $\mathscr{E} \mathscr{H}|k|(Q)$ the corresponding quantum cluster algebra, i.e, the $\mathbb{Z} \mathbb{P}$-subalgebra of $\mathscr{F}$ generated by all the cluster variables. Note that here we are working over a finite field, the definition of quantum cluster algebra in section 2.1 remains valid (see [16]). The main result of this article is the following theorem:

Theorem 1. For any quiver $Q$ of finite type, we have $\mathscr{E} \mathscr{H}_{|k|}(Q)=\mathscr{A} \mathscr{H}_{|k|}(Q)$.

We conjecture that Theorem 1 holds for any quiver of affine type.

Conjecture 1. For any quiver $Q$ of affine type, we have $\mathscr{E} \mathscr{H}_{|k|}(Q)=\mathscr{A}_{|k|}(Q)$.

\section{Proof of the main theorem}

In this section, we fix a quiver $Q$ of finite type with $n$ vertices. Firstly, we recall some notations. For any $k \widetilde{Q}$-modules $M, N$ and $E$, denote by $\varepsilon_{M N}^{E}$ the cardinality of the set $\operatorname{Ext}_{k \widetilde{Q}}^{1}(M, N)_{E}$ which is the subset of $\operatorname{Ext}_{k \widetilde{Q}}^{1}(M, N)$ consisting of those equivalence classes of short exact sequences with middle term isomorphic to $E$ ([14, Section 4$])$. Let $F_{A B}^{M}$ be the number of submodules $U$ of $M$ such that $U$ is isomorphic to $B$ and $M / U$ is isomorphic to $A$. Then by definition, we have

$$
\left|\operatorname{Gr}_{\underline{e}}(M)\right|=\sum_{A, B ; \underline{\operatorname{dim}} B=\underline{e}} F_{A B}^{M} .
$$

Denote by $[M, N]^{1}=\operatorname{dim}_{k} \operatorname{Ext}_{k \widetilde{Q}}^{1}(M, N)$ and $[M, N]=\operatorname{dim}_{k} \operatorname{Hom}_{k \widetilde{Q}}(M, N)$. The following Theorem 2 proved in [7] and Proposition 11 give the explicit relations between $X_{N} X_{M}$ and $X_{N \oplus M}$.

Theorem 2. [7] Let $M$ and $N$ be $k Q$-modules. Then

$$
q^{[M, N]^{1}} X_{M} X_{N}=q^{\frac{1}{2} \Lambda\left(\left(\widetilde{I}-\widetilde{R}^{t r}\right) \underline{m},\left(\widetilde{I}-\widetilde{R}^{t r}\right) \underline{n}\right)} \sum_{E} \varepsilon_{M N}^{E} X_{E} .
$$

Let $M$ be any $k Q$-module and $I$ any injective $k \widetilde{Q}$-module. Define

$$
\operatorname{Hom}_{k \widetilde{Q}}(M, I)_{B I^{\prime}}:=\left\{f: M \longrightarrow I \mid k e r f \cong B, \text { coker } f \cong I^{\prime}\right\} .
$$

Note that $I^{\prime}$ is an injective $k \widetilde{Q}$-module. The following result, together with Theorem 2, is essential for us to prove Theorem 1.

Proposition 1. With the above notations, we have

$$
q^{[M, I]} X_{M} X_{I[-1]}=q^{\frac{1}{2} \Lambda\left(\left(\widetilde{I}-\widetilde{R}^{t r}\right) \underline{m},-\underline{\operatorname{dim}} s o c I\right)} \sum_{B, I^{\prime}}\left|\operatorname{Hom}_{k \widetilde{Q}}(M, I)_{B I^{\prime}}\right| X_{B \oplus I^{\prime}[-1]} .
$$


Proof. We calculate

$$
\begin{aligned}
& X_{M} X_{I[-1]} \\
& =\sum_{G, H} q^{-\frac{1}{2}\langle H, G\rangle} F_{G H}^{M} X^{-\widetilde{B} \underline{h}-\left(\widetilde{I}-\widetilde{R}^{t r}\right) \underline{m}} X^{\underline{\operatorname{dim}} s o c I} \\
& =\sum_{G, H} q^{-\frac{1}{2}\langle H, G\rangle} F_{G H}^{M} q^{\frac{1}{2} \Lambda\left(-\widetilde{B} \underline{h}-\left(\widetilde{I}-\widetilde{R}^{t r}\right) \underline{m}, \underline{\operatorname{dim}} s o c I\right)} X^{-\widetilde{B} \underline{h}-\left(\widetilde{I}-\widetilde{R}^{t r}\right) \underline{m}+\underline{\operatorname{dim} s o c I}} \\
& =q^{\frac{1}{2} \Lambda\left(-\left(\widetilde{I}-\widetilde{R}^{t r}\right)\right.} \underline{m}, \underline{\operatorname{dim} s o c I)} \sum_{G, H} q^{-\frac{1}{2}\langle H, G\rangle} q^{\frac{1}{2} \Lambda(-\widetilde{B} \underline{h}, \underline{\operatorname{dim}} s o c I)} F_{G H}^{M} X^{-\widetilde{B} \underline{h}-\left(\widetilde{I}-\widetilde{R}^{t r}\right)} \underline{m}+\underline{\operatorname{dim} s o c I}
\end{aligned}
$$

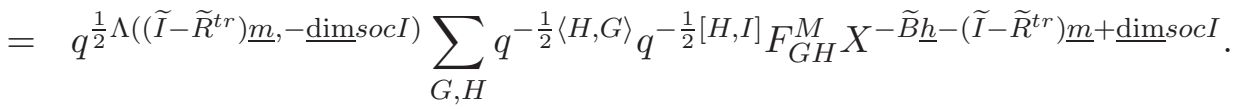

Here we use the fact that

$$
\Lambda(-\widetilde{B} \underline{h}, \underline{\operatorname{dim}} s o c I)=-\underline{h}^{t r} \widetilde{B}^{t r} \Lambda(\underline{\operatorname{dim}} s o c I)=-\underline{h}^{\text {tr }}(\underline{\operatorname{dim}} s o c I)=-[H, I] .
$$

Note that if we have the following commutative diagram

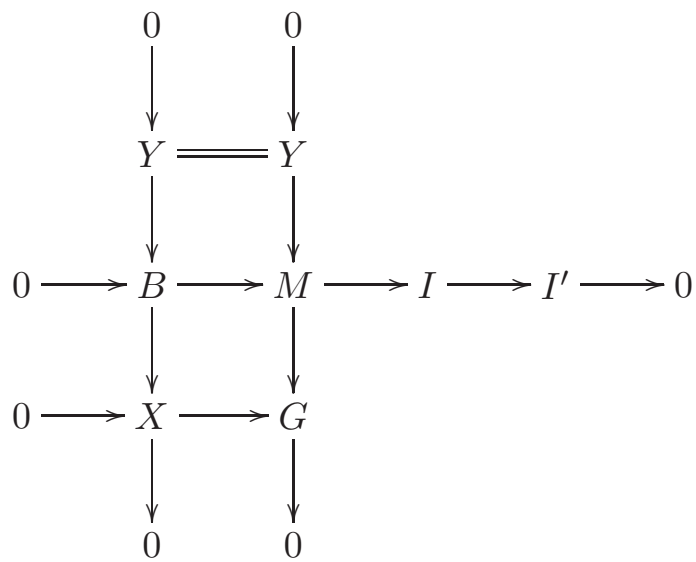

and short exact sequences

$$
\begin{aligned}
& 0 \longrightarrow B \longrightarrow M \longrightarrow A \longrightarrow 0 \\
& 0 \longrightarrow A \longrightarrow I \longrightarrow I^{\prime} \longrightarrow 0,
\end{aligned}
$$

then by [14] it follows that

$$
\sum_{B} F_{X Y}^{B} F_{A B}^{M}=\sum_{G} F_{A X}^{G} F_{G Y}^{M},\left|\operatorname{Hom}_{k \widetilde{Q}}(M, I)_{B I^{\prime}}\right|=\sum_{A}|\operatorname{Aut}(A)| F_{A B}^{M} F_{I^{\prime} A}^{I}
$$

and

$$
\sum_{A, I^{\prime}, X}|\operatorname{Aut}(A)| F_{I^{\prime} A}^{I} F_{A X}^{G}=\sum_{I^{\prime}, X}\left|\operatorname{Hom}_{k \widetilde{Q}}(G, I)_{X I^{\prime}}\right|=q^{[G, I]}=q^{\langle G, I\rangle} .
$$


By [14, Lemma 1], we have $\left(\widetilde{I}-\widetilde{R}^{t r}\right) \underline{i}=\underline{\operatorname{dim}} \operatorname{socI}$. Now we can calculate the term

$$
\begin{aligned}
& \sum_{B, I^{\prime}}\left|\operatorname{Hom}_{k \widetilde{Q}}(M, I)_{B I^{\prime}}\right| X_{B \oplus I^{\prime}[-1]} \\
= & \sum_{A, B, I^{\prime}, X, Y}|\operatorname{Aut}(A)| F_{A B}^{M} F_{I^{\prime} A}^{I} q^{-\frac{1}{2}\left\langle Y, X-I^{\prime}\right\rangle} F_{X Y}^{B} X^{-\widetilde{B} \underline{y}-\left(\widetilde{I}-\widetilde{R}^{t r}\right) \underline{b}+\underline{\operatorname{dim} s o c I^{\prime}}} \\
= & \sum_{A, G, I^{\prime}, X, Y} q^{-\frac{1}{2}\left\langle Y, X-I^{\prime}\right\rangle}|\operatorname{Aut}(A)| F_{I^{\prime} A}^{I} F_{A X}^{G} F_{G Y}^{M} X^{-\widetilde{B} \underline{y}-\left(\widetilde{I}-\widetilde{R}^{t r}\right) \underline{b}+\underline{\operatorname{dim} s o c I^{\prime}} .}
\end{aligned}
$$

Note that we have the following facts

$$
\underline{i}^{\prime}+\underline{a}=\underline{i}, \underline{x}+\underline{a}=\underline{g} \Longrightarrow \underline{x}-\underline{i}^{\prime}=\underline{g}-\underline{i},
$$

and

$$
\begin{aligned}
& -\widetilde{B} \underline{y}-\left(\widetilde{I}-\widetilde{R}^{t r}\right) \underline{b}+\underline{\operatorname{dim}} s o c I^{\prime} \\
= & -\widetilde{B} \underline{h}-\left(\widetilde{I}-\widetilde{R}^{t r}\right)\left(\underline{m}-\underline{i}-\underline{i}^{\prime}\right)+\underline{\operatorname{dim}} s o c I^{\prime} \\
= & -\widetilde{B} \underline{h}-\left(\widetilde{I}-\widetilde{R}^{t r}\right) \underline{m}+\left(\widetilde{I}-\widetilde{R}^{t r}\right)\left(\underline{i}-\underline{i}^{\prime}\right)+\underline{\operatorname{dim} s o c I^{\prime}} \\
= & -\widetilde{B} \underline{h}-\left(\widetilde{I}-\widetilde{R}^{t r}\right) \underline{m}+\left(\widetilde{I}-\widetilde{R}^{t r}\right) \underline{i} \\
= & -\widetilde{B} \underline{h}-\left(\widetilde{I}-\widetilde{R}^{t r}\right) \underline{m}+\underline{\operatorname{dim}} \operatorname{soc} I .
\end{aligned}
$$

Hence

$$
\begin{aligned}
& \sum_{B, I^{\prime}}\left|\operatorname{Hom}_{k \widetilde{Q}}(M, I)_{B I^{\prime}}\right| X_{B \oplus I^{\prime}[-1]} \\
= & \sum_{G, H} q^{\langle G, I\rangle} q^{-\frac{1}{2}\langle H, G-I\rangle} F_{G H}^{M} X^{-\widetilde{B} \underline{h}-\left(\widetilde{I}-\widetilde{R}^{t r}\right) \underline{m}+\underline{\operatorname{dim}} s o c I} \\
= & \sum_{G, H} q^{\langle M, I\rangle} q^{-\frac{1}{2}\langle H, I\rangle} q^{-\frac{1}{2}\langle H, G\rangle} F_{G H}^{M} X^{-\widetilde{B} \underline{h}-\left(\widetilde{I}-\widetilde{R}^{t r}\right) \underline{m}+\underline{\operatorname{dim} s o c I}} \\
= & q^{[M, I]} \sum_{G, H} q^{-\frac{1}{2}[H, I]} q^{-\frac{1}{2}\langle H, G\rangle} F_{G H}^{M} X^{-\widetilde{B} \underline{h}-\left(\widetilde{I}-\widetilde{R}^{t r}\right) \underline{m}+\underline{\operatorname{dim}} s o c I} .
\end{aligned}
$$

This finishes the proof.

Remark 1. Proposition 1 holds for any acyclic quiver.

The following lemma is well-known. Here we give a sketch of the proof following [5, Lemma 8(b)].

Lemma 1. Let

$$
M \longrightarrow E \longrightarrow N \stackrel{\epsilon}{\rightarrow} M[1]
$$

be a non-split triangle in $\mathscr{C}_{\widetilde{Q}}$. Then

$$
\operatorname{dim}_{k} \operatorname{Ext}_{\mathscr{C}_{\widetilde{Q}}}^{1}(E, E)<\operatorname{dim}_{k} \operatorname{Ext}_{\mathscr{C}_{\widetilde{Q}}}^{1}(M \oplus N, M \oplus N) .
$$


Proof. For any object $L \in \mathscr{C}_{\widetilde{Q}}$, applying the functor $\operatorname{Ext}_{\mathscr{C}_{\widetilde{Q}}}^{1}(-, L)$ to the above non-split triangle gives rise to the exact sequence

$0 \longrightarrow \operatorname{kerf}_{L} \longrightarrow \operatorname{Ext}_{\mathscr{C}_{\tilde{Q}}}^{1}(N, L) \stackrel{f_{L}}{\longrightarrow} \operatorname{Ext}_{\mathscr{C}_{\tilde{Q}}}^{1}(E, L) \stackrel{g_{L}}{\longrightarrow} \operatorname{Ext}_{\mathscr{C}_{\tilde{Q}}}^{1}(M, L) \longrightarrow \operatorname{cokerg}_{L} \longrightarrow 0$

Thus we have

$\operatorname{dim}_{k} \operatorname{kerf}_{L}+\operatorname{dim}_{k} \operatorname{Ext}_{\mathscr{C}_{\tilde{Q}}}^{1}(E, L)+\operatorname{dim}_{k} \operatorname{cokerg}_{L}=\operatorname{dim}_{k} \operatorname{Ext}_{\mathscr{C}_{\tilde{Q}}}^{1}(N, L)+\operatorname{dim}_{k} \operatorname{Ext}_{\mathscr{C}_{\tilde{Q}}}^{1}(M, L)$

Hence

$$
\begin{aligned}
& \operatorname{dim}_{k} \operatorname{Ext}_{\mathscr{C}_{\widetilde{Q}}}^{1}(E, N) \leq \operatorname{dim}_{k} \operatorname{Ext}_{\mathscr{C}_{\widetilde{Q}}}^{1}(N, N)+\operatorname{dim}_{k} \operatorname{Ext}_{\mathscr{C}_{\widetilde{Q}}}^{1}(M, N) \\
& \operatorname{dim}_{k} \operatorname{Ext}_{\mathscr{C}_{\widetilde{Q}}}^{1}(E, E) \leq \operatorname{dim}_{k} \operatorname{Ext}_{\mathscr{C}_{\widetilde{Q}}}^{1}(N, E)+\operatorname{dim}_{k} \operatorname{Ext}_{\mathscr{C}_{\widetilde{Q}}}^{1}(M, E) .
\end{aligned}
$$

Note that $0 \neq \epsilon \in \operatorname{ker} f_{M}$, so we have

$$
\operatorname{dim}_{k} \operatorname{Ext}_{\mathscr{C}_{\widetilde{Q}}}^{1}(E, M)<\operatorname{dim}_{k} \operatorname{Ext}_{\mathscr{C}_{\widetilde{Q}}}^{1}(N, M)+\operatorname{dim}_{k} \operatorname{Ext}_{\mathscr{C}_{\widetilde{Q}}}^{1}(M, M) .
$$

Therefore

$$
\begin{aligned}
\operatorname{dim}_{k} \operatorname{Ext}_{\mathscr{C}_{\widetilde{Q}}}^{1}(M \oplus N, M \oplus N) & >\operatorname{dim}_{k} \operatorname{Ext}_{\mathscr{C}_{\widetilde{Q}}}^{1}(E, N)+\operatorname{dim}_{k} \operatorname{Ext}_{\mathscr{C}_{\widetilde{Q}}}^{1}(E, M) \\
& =\operatorname{dim}_{k} \operatorname{Ext}_{\mathscr{C}_{\widetilde{Q}}}^{1}(N, E)+\operatorname{dim}_{k} \operatorname{Ext}_{\mathscr{C}_{\tilde{Q}}}^{1}(M, E) \\
& \geq \operatorname{dim}_{k} \operatorname{Ext}_{\mathscr{C}_{\tilde{Q}}}^{1}(E, E) .
\end{aligned}
$$

This proves our assertion.

Proof of Theorem [1: We need to prove that for any cluster character $X_{L} \in$ $\mathscr{A} \mathscr{H}_{|k|}(Q)$, then $X_{L} \in \mathscr{E} \mathscr{H}_{|k|}(Q)$.

Let $L \cong \bigoplus_{i=1}^{l} L_{i}^{\oplus n_{i}}, n_{i} \in \mathbb{N}$ where $L_{i}(1 \leq i \leq l)$ are indecomposable objects in $\mathscr{C}_{\widetilde{Q}}$. Thus $X_{L_{i}}(1 \leq i \leq l)$ are in $\mathscr{E} \mathscr{H}_{|k|}(Q)$. By Theorem 2, Proposition [ and Lemma 1, we have that

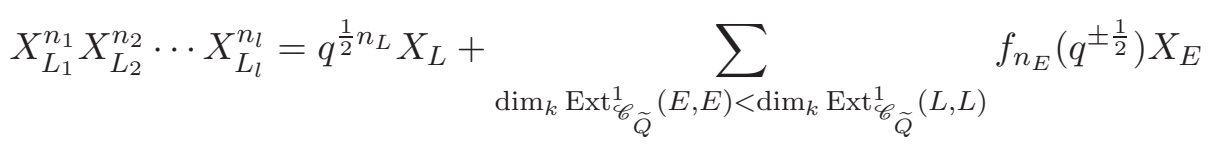

where $n_{L} \in \mathbb{Z}$ and $f_{n_{E}}\left(q^{ \pm \frac{1}{2}}\right) \in \mathbb{Z}\left[q^{ \pm \frac{1}{2}}\right]$. Thus by induction, we can prove that $X_{L} \in \mathscr{E} \mathscr{H}_{|k|}(Q)$ which implies $\mathscr{E} \mathscr{H}_{|k|}(Q)=\mathscr{A} \mathscr{H}_{|k|}(Q)$.

Acknowledgements The author would like to thank Professor Jie Xiao, Doctor Fan Xu and Doctor Jie Sheng for very helpful conversations.

\section{References}


1. Berenstein A, Fomin S, Zelevinsky A. Cluster algebras III: Upper bounds and double Bruhat cells. Duke Math. J., 2005, 126: 1-52

2. Buan A, Marsh R, Reineke M, Reiten I, Todorov G. Tilting theory and cluster combinatorics. Adv. Math., 2006, 204: 572-618

3. Berenstein A, Zelevinsky A. Quantum cluster algebras. Adv. Math., 2005, 195: 405455

4. Caldero P, Chapoton F. Cluster algebras as Hall algebras of quiver representations. Comm. Math. Helv., 2006, 81: 595-616

5. Caldero P, Keller B. From triangulated categories to cluster algebras. Invent. Math., 2008, 172(1): 169-211

6. Ding M, Xu F. Bases of the quantum cluster algebra of the Kronecker quiver. arXiv:1004. $2349 \mathrm{v} 4$ [math.RT]

7. Ding $\mathrm{M}, \mathrm{Xu} \mathrm{F}$. The multiplication theorem and bases in finite and affine quantum cluster algebras. arXiv:1006.3928v3 [math.RT]

8. Ding M, Xiao J, Xu F. Integral bases of cluster algebras and representations of tame quivers. arXiv:0901.1937 [math.RT]

9. Fomin S, Zelevinsky A. Cluster algebras. I. Foundations. J. Amer. Math. Soc., 2002, 15(2): 497-529

10. Fomin S, Zelevinsky A. Cluster algebras. II. Finite type classification. Invent. Math., 2003, 154(1): 63-121

11. Geiss C, Leclerc B, Schröer J. Kac-Moody groups and cluster algebras. arXiv:1001.3545 v2 [math.RT]

12. Geiss C, Leclerc B, Schröer J. Generic bases for cluster algebras and the Chamber Ansatz. arXiv:1004.2781v2 [math.RT]

13. Grabowski J, Launois S. Quantum cluster algebra structures on quantum Grassmannians and their quantum Schubert cells: the finite-type cases. Int Math Res Notices, 2010, doi: 10.1093/imrn/rnq153

14. Hubery A. Acyclic cluster algebras via Ringel-Hall algebras. preprint, 2005, available at the author's homepage

15. Lampe P. A quantum cluster algebra of Kronecker type and the dual canonical basis. Int Math Res Notices, 2010, doi: 10.1093/imrn/rnq162

16. Qin F. Quantum cluster variables via Serre polynomials. arXiv:1004.4171v2 [math.QA]

17. Rupel D. On a quantum analogue of the Caldero-Chapoton Formula. Int Math Res Notices, 2010, doi:10.1093/imrn/rnq192 